A simple method of producing far-infrared high-pass filters

This article has been downloaded from IOPscience. Please scroll down to see the full text article.

1991 Meas. Sci. Technol. 2203

(http://iopscience.iop.org/0957-0233/2/3/003)

View the table of contents for this issue, or go to the journal homepage for more

Download details:

IP Address: 132.199.32.109

The article was downloaded on 11/10/2010 at 14:20

Please note that terms and conditions apply. 


\title{
A simple method of producing far-infrared high-pass filters
}

\author{
P G Huggard†, Gi Schneider $\neq$, W Prett $\mid \neq$ and W Blau $†$ \\ † Department of Pure and Applied Physics, Trinity College, Dublin 2, \\ Republic of Ireland \\ ‡Institut für Angewandte Physik, Universität Regensburg, 8400 Regensburg, \\ Federal Republic of Germany
}

Received 23 October 1989, in final form 21 September 1990, accepted for publication 8 November 1990

\begin{abstract}
A method of making high-pass waveguide filters for the far infrared is described. The filters are formed from arrays of tightly packed narrow-bore metal tubes. Such filters feature sharp cut-off transitions in the region between $20 \mathrm{~cm}^{-1}$ and $40 \mathrm{~cm}^{-1}$. Transmission above cut-off is comparable to that in similar filters produced by drilling holes in thin metal sheets. Typically the transmissions just above cut-off are about $5 \%$ while that just below was measured as less than $0.1 \%$.
\end{abstract}

\section{Introduction}

A problem encountered in the detection of far infrared/ submillimetre radiation is that detectors commonly used (Golay cells, pyroelectric and bolometric devices) have a broad spectral response. Thus pre-detector filtering is necessary if a restricted spectral response is required e.g. for the detection of second- and third-harmonic radiation generated by non-linear optical processes (Mayer and Keilmann 1986a, b). High (frequency) pass filters were developed for use in this spectral region (Keilmann 1981). These are arrays of cylindrical waveguides produced by manually drilling metal foils. Such filters feature high (but non-uniform) transmission in the pass region with a sharp transition at the cut-off frequency. Transmission below cut-off may be made arbitrarily small by increasing the length of the filter.

The governing factor in the choice of a harmonic separation filter is not the passband transmission but the contrast between transmissions above and below cut-off. Conventional filtering techniques for the 10 to $50 \mathrm{~cm}^{-1}$ region, e.g. inductive metal meshes (Renk and Genzel 1962) or annular slot arrays (Krug et al 1989), have high-pass band transmissions. Both, however, suffer from a gradual cut-on of transmission and are thus not suitable for harmonic separation. Thus a hole filter with, say, $10 \%$ transmission above cut-off and $10^{-6} \%$ below (Keilmann 1981) is the only practical choice.

For a cylindrical guide with internal diameter $d$ the cut-off frequency $v_{\mathfrak{c}}$ for the mode with the lowest cutoff frequency $\left(\mathrm{TE}_{11}\right)$, is given by (Keilmann 1981)

$$
v_{\mathrm{c}}=\frac{5.86 \mathrm{~cm}^{-1}}{d}
$$

with $d$ measured in $\mathrm{mm}$. This assumes that the guide is filled with a lossless dielectric with a refractive index of unity. Thus to make guides with $v_{\mathrm{c}}$ in the $20-40 \mathrm{~cm}^{-1}$ range, where strong laser lines are available, holes with internal diameters between 300 and $150 \mu \mathrm{m}$ must be formed. The fabrication of an array of some hundreds of such holes by mechanical drilling is a difficult and time-consuming task, requiring precision equipment and much skill. An additional problem is that the fractional variation of diameter amongst the holes increases as the diameter is reduced (Keilmann 1981), leading to a reduction in the sharpness of cut-off governed by the precision of drilling.

We report below on an improved method of manufacture of waveguide filters. Our filters were made by forming bundles from small tubes and then slicing the bundles transversely to form filters of the required thickness. This method requires no special apparatus or skill and allows the manufacture of filters up to at least $1 \mathrm{~mm}$ thickness. The use of preformed tubing, with regular bores, gives a sharp cut-off. Transmission spectra for three such filters are presented below and show that cut-off frequencies are predicted to within $5 \%$ using equation (1).

\section{Filter construction}

Stainless steel was chosen as the tube material because of its mechanical strength and resistance to corrosion. Additionally it is readily available in several diameters and wall thicknesses as the raw material for hypodermic needles. Three gauges of tubing were obtained from Tomlinson Tube \& Instrument Ltd, Bidford on Avon, UK. Table 1 presents properties of the tubing and filters. The tubing was cut into $10 \mathrm{~mm}$ lengths and thoroughly 
Table 1. Characteristics of tubing and filters.

\begin{tabular}{llll}
\hline Filter & A & B & C \\
\hline Filter aperture $(\mathrm{mm})$ & 5.16 & 6.0 & 5.00 \\
No of tubes & 110 & 275 & 320 \\
$\begin{array}{l}\text { Average internal diameter } \\
\quad \text { of tube }(\mathrm{mm})\end{array}$ & 0.213 & 0.182 & 0.139 \\
\begin{tabular}{l} 
Filter thickness $(\mathrm{mm})$ \\
\hline
\end{tabular} & 0.650 & 0.450 & 0.370 \\
\hline
\end{tabular}

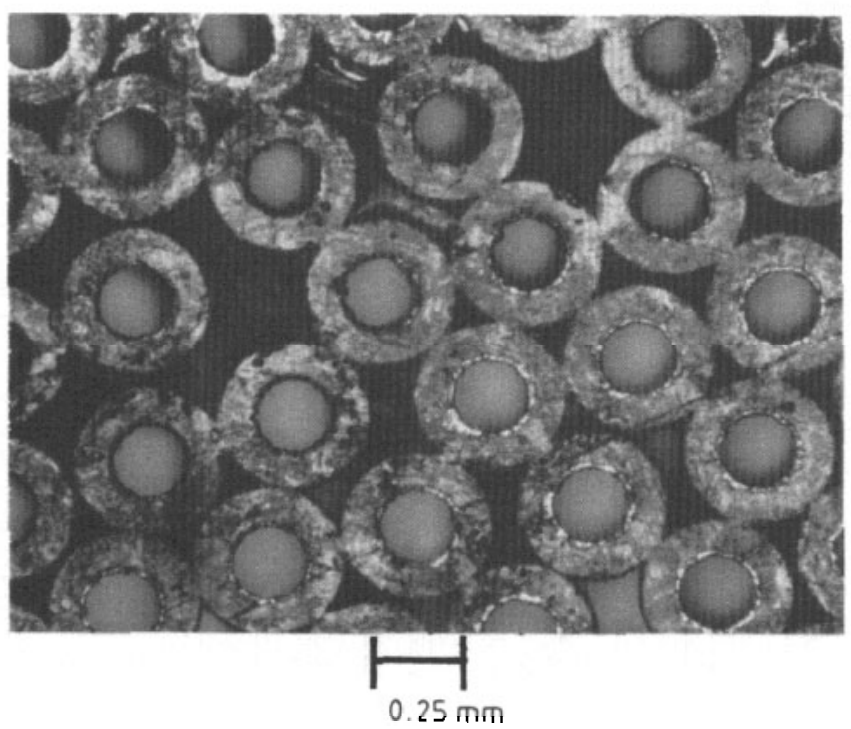

Figure 1. Micrograph of filter A. Average hole size is $0.213 \mathrm{~mm}$.

degreased with laboratory detergent. The cut lengths were tightly packed into holes drilled $8 \mathrm{~mm}$ deep into the end of a brass rod. Molten 60:40 Pb: Sn solder, used with a flux for the soft soldering of stainless steel ('Frysol', Fry's Metal Ltd, Merton Abbey, London, UK), was sufficiently fluid to fill the interstices between the tubes. Soldered bundles were cut transversely with a low-speed diamond saw and the cut surfaces were polished on 1200-grade emery paper.

The individual guides, which become blocked with detritus during cutting and polishing, were then cleaned manually while viewing the filter through a microscope. The filters were further cleaned in an ultrasonic bath and inspected for voids. Any defects larger than the tube diameter were repaired by the application of a small quantity of silver-loaded paint. Micrographs of parts of filters $A$ and $C$ are shown in figures 1 and 2 .

\section{Transmission and attenuation measurements}

Transmission spectra of the filters $A, B$ and $C$ were taken using a commercial Fourier transform spectrometer, comparing the filter with an aperture of the same area in each case. The spectra are shown in figure 3 , with a close-up of the cut-off region in figure 4 . No

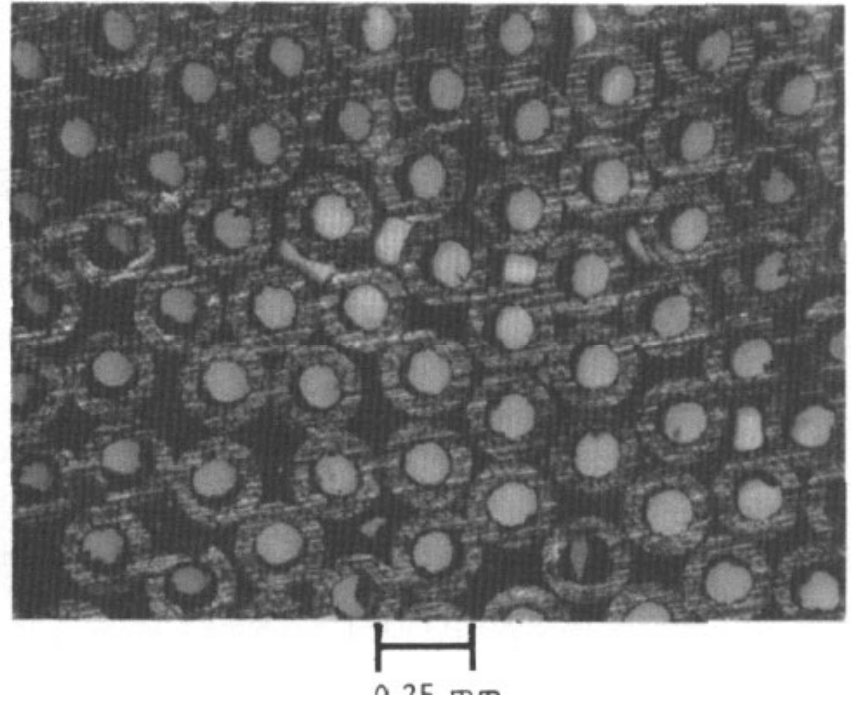

Figure 2. Micrograph of filter C. Average hole size is $0.139 \mathrm{~mm}$. The variation amongst holes is greater than A, reducing the sharpness of cut-off.

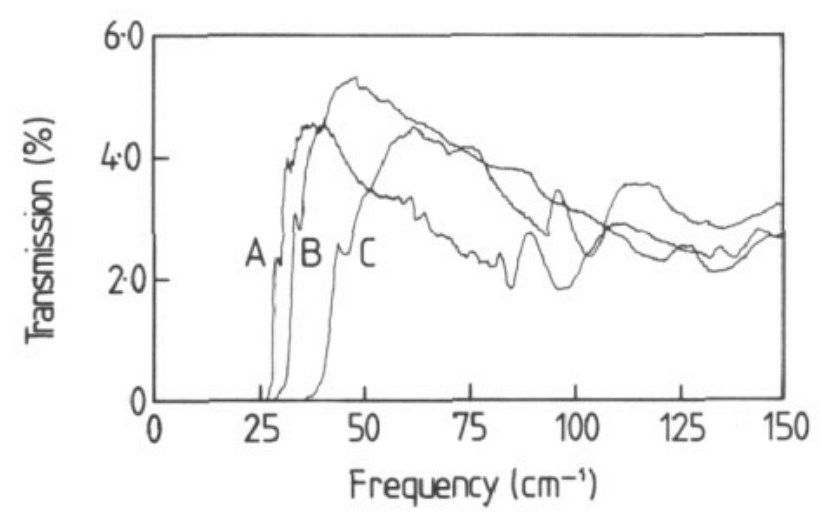

Figure 3. Fourier transform transmission spectra of filters $\mathrm{A}, \mathrm{B}$ and $\mathrm{C}$. Spectrometer resolution is $0.2 \mathrm{~cm}^{-1}$.

attempt was made to measure the reflectance of the filters.

The cut-off frequency was measured in each case by ignoring the low-frequency tail of the transmission curve, figure 4 , and continuing the linear part of the curve to the $x$-axis. Results are listed in table 2 along with theoretical cut-offs calculated from equation (1) using the measured average tube diameter. The value of cutoff frequency defined by equation (1) is that frequency below which the guided wave vector becomes purely imaginary, giving an exponential decay of power down the guide. Agreement between calculated and measured cut-offs is excellent for filters $A$ and $B$. However the experimental cut-off is about $2 \mathrm{~cm}^{-1}$ below the expected value for filter $C$. The reason for this shift can be explained by examining the micrographs. The holes in $C$ are more uneven and distorted from circular than those of $A$, thus allowing the propagation of modes with 


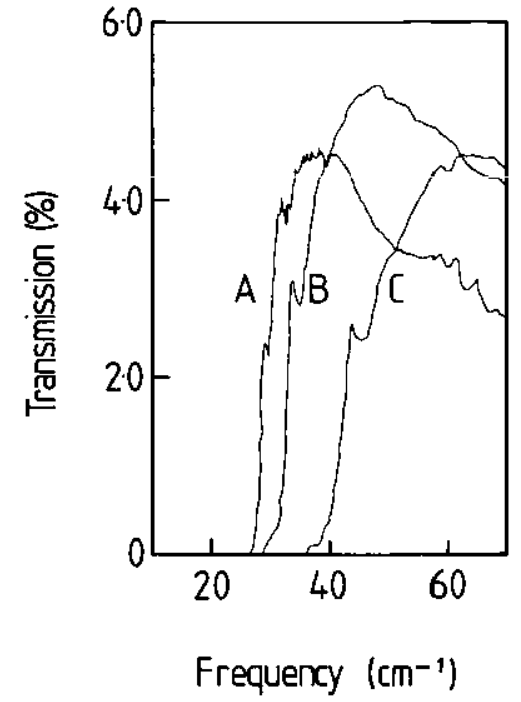

Figure 4. Expanded view of the transmission close to cut-off of filters A, B and C. Extrapolated cut-off frequencies are $27.5,32$ and $40 \mathrm{~cm}^{-1}$ respectively. Resolution $=0.2 \mathrm{~cm}^{-1}$.

Table 2. Comparison between theoretical and measured cut-off frequencies.

\begin{tabular}{llll}
\hline Filter. & A & B & C \\
\hline Theoretical cut-off frequency $\left(\mathrm{cm}^{-1}\right)$ & 27.5 & 32.2 & 42.1 \\
Measured cut-off frequency $\left(\mathrm{cm}^{-1}\right)$ & 27.5 & 32 & 40 \\
\hline
\end{tabular}

lower cut-off frequencies than those predicted from the average diameter.

Peak transmissions at the first maximum above cutoff are about $5 \%$ for all three filters. In the case of filter A this compares favourably with a peak transmission of 3.7\% reported (Mayer and Keilmann 1986a) for a drilled filter $1.03 \mathrm{~mm}$ thick but with similar hole sizes and separation. It is thought that several factors account for the low peak transmission, the first being the fractional area of the holes. If the tubes used for our filters were hexagonally closely packed these areas (neglecting edge effects) would be 23,25 and $32 \%$ for A, B and C respectively: achieved ratios were approximately 0.8 of these. A very strong dependence of peak transmission on packing density of holes is reported (Mayer and Keilmann 1986a) with peak transmission increasing by a factor of 20 for a fourfold increase in density.

Secondly, a rapid drop in transmission is observed (Keilmann 1981) as the angle of incidence of the radiation deviates from the normal: the power transmitted dropped by $50 \%$ when the filter was tilted by $7^{\circ}$. Thus any slight variation in angles between the individual tubes within the bundle seriously affects the overall transmission. Finally the attenuation of guided radiation may be high, due to dirt, scratches and possible deviations from a circular cross section.

To measure attenuation of the filters above the cut-

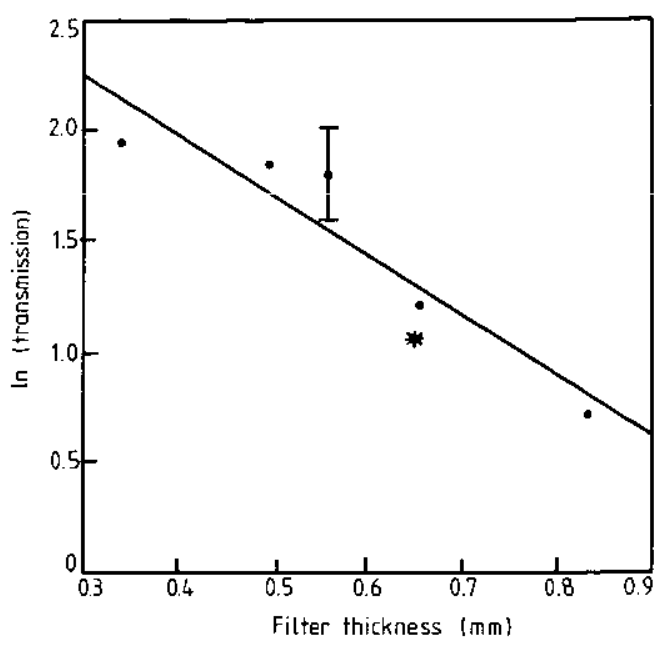

Figure 5. Plot of In (transmission) against filter thickness, for filters with the same tube pattern as filter A. Points are from FIR laser measurements except for the point indicated by ${ }^{*}$, which is from the Fourier spectrometer curve.

off frequency four filters were cut from the same bundle as filter $\mathrm{A}$, giving filters with the same hole pattern but with thicknesses varying between $0.34 \pm 0.01 \mathrm{~mm}$ and $0.83 \pm 0.01 \mathrm{~mm}$. Attenuation was measured at a single frequency of $84 \mathrm{~cm}^{-1}(\lambda=118 \mu \mathrm{m})$. The source of this radiation was a conventional $\mathrm{CH}_{3} \mathrm{OH}$ vapour FIR laser, optically pumped by a $\mathrm{CW} \mathrm{CO}_{2}$ laser (Edinburgh Instruments $295 /$ PL5 system). To obtain a reference the beam was divided by a $6 \mu \mathrm{m}$ mylar film placed at $45^{\circ}$ to the direction of propagation. The diverted radiation was focused with a TPX lens onto an Eltec-400 pyroelectric detector. The remainder of the $10 \mathrm{~mm}$ diameter laser beam was focused by a $30 \mathrm{~cm}$ focal length off-axis parabolic mirror onto a similar detector, giving a spot size of about $5 \mathrm{~mm}$. The filters were placed $12 \mathrm{~mm}$ from the surface of the latter detector. Signals from the detectors were averaged with lock-in amplifiers.

The results of these measurements are shown in figure 5 together with an additional point, obtained from the Fourier spectrum, which is included to show the agreement between results. A best-fit line to the points is shown, giving a power absorption coefficient of $3.1 \mathrm{~mm}^{-1}$. Thus the propagation losses at frequencies approximately twice cut-off are of the same magnitude as losses coupling the radiation into the guides.

In a similar manner to above cut-off, the attenuation just below was measured for filter $\mathrm{B}$, at a frequency of $22.4 \mathrm{~cm}^{-1}$. The laser medium in this case was $\mathrm{CH}_{3} \mathrm{I}$ vapour. The transmission at this frequency was measured as $7 \pm 3 \times 10^{-2 \%} \%$, showing the excellent contrast between stop and pass bands.

\section{Conctusions}

The feasibility of constructing far-infrared high-pass filters from bundles of individual tubes has been established. Such filters have proved easy to make, and give 
a sharp cut-off. Peak transmissions are comparable to those of similar filters produced by drilling. However, considerable improvement in transmission would be obtained if thinner-walled tubing was used, thus decreasing the interhole spacing. It is expected that transmission would also rise if the surface quality of the interior of the guides was improved, either by utilising better quality tubing or by chemical polishing.

It is also feasible to make filters from tubing of differing bores - this should allow the fabrication of filters with gradually increasing transmissions governed by the mix of tubes. Such a filter, if used in conjunction with a restrahlen crystal, would form a far-infrared bandpass filter.

\section{References}

Keilmann F 1981 Infrared high-pass filter with high contrast Int. J. Infrared and Millimeter Waves 2 259-72

Krug P A, Davies D H, McPhedran R C, Wright W, Macfarlane J C and Whitbourn L B 1989 Annular-slot arrays as far-infrared bandpass filters Opt. Lett. 14 931-3

Mayer A and Keilmann F 1986a Far-infrared nonlinear optics. I. $\chi^{(2)}$ near ionic resonance Phys. Rev. B 33 6954-61

1986b Far-infrared nonlinear optics. II. $\chi^{(3)}$ contributions from the dynamics of free carriers in semiconductors Phys. Rev. B 33 6962-8

Renk K F and Genzel L 1962 Interference filters and FabryPerot interferometers for the far infrared Appl. Opt. 1 643-8. 